\title{
Individualism and Partnership: A Descriptive Qualitative Analysis of the Chronic Disease Phenomenon as Perceived by Older Adults
}

\author{
Kimberly Sell1, Elaine Amella1, Martina Mueller ${ }^{1}$, Jeannette Andrews ${ }^{1}$, Joy Wachs ${ }^{2}$ \\ ${ }^{1}$ Medical University of South Carolina, Charleston, USA \\ ${ }^{2}$ East Tennessee State University, Johnson City, USA \\ Email: sell@etsu.edu, amellaej@musc.edu, muellermm@musc.edu, JANDREWS@mailbox.sc.edu, \\ WACHS@mail.etsu.edu,
}

Received 23 September 2015; accepted 26 October 2015; published 29 October 2015

Copyright (C) 2015 by authors and Scientific Research Publishing Inc.

This work is licensed under the Creative Commons Attribution International License (CC BY). http://creativecommons.org/licenses/by/4.0/

(c) (i) Open Access

\begin{abstract}
With the older adult population projected to increase substantially in the next 10 years, the incidence of chronic disease will become a significant factor in the burden of disease both globally and within the United States. The chronic disease state has been shown to decrease quality of life and the life expectancy of those individuals. In Tennessee the incidence of chronic disease, lower incomes, and education levels increases the risk of decreased quality of life and increased mortality in the older adult population. A review of literature by the researcher reveals that scant research has focused upon the older adult's perspective toward chronic illness and making changes to their health routine. As part of a mixed-method correlational study, focus groups used a descriptive qualitative approach to increase understanding of the phenomenon of chronic illness. The purpose of this qualitative study was to determine the attitudes and perceptions of older adults in East Tennessee towards behavior change and health maintenance in chronic disease.
\end{abstract}

Keywords

Qualitative, Chronic Disease, Older Adults

\section{Introduction}

Due to changes in medical care, the older adult population is living longer and the proportion of older adults will

How to cite this paper: Sell, K., Amella, E., Mueller, M., Andrews, J. and Wachs, J. (2015) Individualism and Partnership: A Descriptive Qualitative Analysis of the Chronic Disease Phenomenon as Perceived by Older Adults. Open Journal of Nursing, 5, 935-947. http://dx.doi.org/10.4236/ojn.2015.510099 
continue to grow until one in every five Americans will be over the age of 65 by the year 2050 [1]. While life expectancy is increasing, the number of chronic diseases also continues to grow necessitating further understanding of the chronic disease phenomenon as perceived by the older adult. The aim of this current study is to develop an understanding of the perceptions of older adults towards the chronic disease phenomenon using a descriptive qualitative method. This study is part of a mixed-method study in which 29 older adults from Northeast Tennessee participate in focus groups. The analysis of qualitative data revealed that older adults desired individualized care and wanted to be an active partner in the healthcare team. As the participants felt like this is a current gap in healthcare, knowledge of this desire by older adults can be beneficial to clinical approaches in chronic disease care.

\subsection{Background}

Chronic disease is a burden for all involved including the individual, family, healthcare provider, and healthcare systems. For individuals with chronic disease, there is a significant risk of premature death, prolonged disability, and an overall decreased quality of life [1] [2]. For those living with chronic disease, 25\% have daily limitations [3]. For healthcare providers, the burden is to deliver care to complex patients with co-morbidities in a cost-effect manner. For example, for individuals with one chronic disease, the average number of physician visits is 13 times per year and this number increases drastically as the number of chronic diseases increases [4]. Healthcare systems struggle to find ways to efficaciously manage patients and control costs. Presently, for those with chronic disease $95 \%$ of healthcare costs are related to healthcare visits [1] and as a result of the increased numbers of older adults, by 2020 the cost will increase by $25 \%$ to a total of 903 billion spent on chronic disease care (CDC, 2013).

Not only are the prevalence and impact of disease changing, but also the inherent characteristics of the population are changing. The silent generations are those who have experienced the great depression and war [5]. These are individuals who, as a result of their experiences, learn to be conservative, frugal, and to focus on community instead of self [5]. For those born between 1946 and 1964, they have become the baby boomer generation. They are a unique generation that contrasts sharply in viewpoints from those generations before them. They have grown and developed during a period of an increased wealth, spending [6], and increased consumerism [6] which is a stark contrast to previous generations. The boomers are more likely to challenge processes, focus more on individuality than collectivism [6], and communicate opinions openly and unreservedly [5]. The baby boomers are a generation of consumers with high expectations and demands, and are less likely to accept physical changes as natural components of aging [6] but instead more likely to seek health interventions to address them. They are the generations who change the expectation of the career purpose from working to provide to one of working towards retirement [6]. As the previous generations are viewed as conformists, who are conservative, respectful of authority and the systems with which they interact [5], the baby boomer population displays significant contrasts through avid consumerism. This dissimilarity with past healthcare consumers results in a healthcare consumer who may very likely change the way that healthcare is delivered and therefore signals an imminent need to understand the perspective of the older adult population towards chronic disease and managing the chronic disease process.

\subsection{Theoretical Framework}

The Social Cognitive Theory is a theoretical framework that encompasses a broad spectrum of characteristics affecting the ability of individuals to change such as setting goals, identifying barriers, and beliefs [7]. This theory supports both internal and external factors for behavior change [8] and provides the structure for the focus group questions. The key concepts within the social cognitive theory are reciprocal determinism, outcome expectations, observational learning, incentive motivation, facilitation and moral disengagement [8]. The social cognitive theory also recognizes the impact that the environment can have on an individual as well as the effect the individual can have on the environment. Reciprocal determinism is the three-way (triadic) interaction among these variables [8] [9]. In this process, the individual, social factors, and the environment facilitate the type of reaction an individual may have in a given situation [9]. Understanding the process of reciprocal determinism in behavior change enhances understanding of potential barriers and social norms of learner self-management [8]. With the Social Cognitive Theory, the individual's expectations and perceptions of both the self-management process and disease status are addressed as well as environmental and social obstacles to changing health behaviors. 


\section{Method}

A qualitative study using focus groups was completed as part of convergent-parallel mixed method study.

\subsection{Study Design}

The descriptive qualitative study was part of a larger mixed method study in which thematic analysis was used to correlate the quantitative data in a convergent-parallel design. The theoretical framework that was used for the study was the Social Cognitive Theory. Participants were recruited from area churches using parish nurses as community liaisons. Those participants were then given lunch and invited to participate in a focus group consisting of 5 - 8 individuals. The individual participated in a focus group at their home church. Prior to beginning the focus group, the participants were instructed about the purpose of the study and consent was obtained. Rules for the group interaction were reviewed before beginning the discussion. The focus group utilized 13 prepared questions (Appendix 1) and was conducted using a semi-structured format that allows for evolution of the conversation as the themes emerge [10]. These questions were derived from the concepts within the Social Cognitive Theory to obtain insights into the perception regarding behavior change, goal attainment, internal and external barriers to change.

The Krueger focus group methodology [11] was used to structure the interaction. The data collection process was completed within the one meeting to reduce burden and attrition of the participants. The interaction was recorded to verify field notes, and field notes were collected by a second member of the research team.

\subsection{Sample}

Qualitative analysis was completed as part of a mixed method study in which focus groups were held using purposive sampling [12] to collect data. Stratified purposive sampling was used in faith based organizations in the Appalachian Region of Northeast Tennessee to recruit older adults diagnosed with diseases reflective of the regional population where chronic disease ranks in the top six for mortality [13]. All individuals who were age 55 (or greater) and had been diagnosed with hypertension, Type 2 diabetes, or chronic obstructive pulmonary disease were included. If the individual had been hospitalized within 30 days prior to the study or non-English speaking, they were excluded. Individuals who had cognitive disorders were also excluded from the study. A total of 29 participants were eligible and enrolled in the study.

\subsection{Ethical Consideration}

The study received approval from the Internal Review Board (IRB) at the Medical University of South Carolina as well as the IRB from East Tennessee State University.

\subsection{Data Collection}

For data collection, 5 focus groups were held consisting of 5 - 8 participants. During the research study, the participant participated in a focus group to evaluate their perceptions of self-management in chronic disease, attitude to self-management and behavior change, as well as barriers and facilitators to the change process. For data collection, a semi-structured discussion format was utilized in the focus groups. The focus group setting allowed for conversation to evolve from multiple perspectives. Data saturation was achieved in each focus group and at that time the focus groups were ended.

\subsection{Analysis}

Once the focus groups concluded, the audio recordings were then transcribed verbatim and compared to the field notes. For qualitative data analysis, the six phase of thematic analysis [14] was used. Substantive coding was used to determined themes in relation to the chosen theoretical framework. The thematic analysis occurred in a sequential fashion with first the data being, transcribed, read, and then re-read by two investigators (KS, JW); once the data had been reviewed twice, the data set was coded and organized into potential themes as determined by the investigators (KS, JW); these themes were then organized into an table for analysis. In addition to theme analysis, coding was accomplished through the use of QRS NVivo 10 software [15]. In addition to coding for identification of prevalent themes [16], the analysis format also supported the purpose of data merging in the 
mixed method feasibility study [17].

\subsection{Qualitative Rigor}

The rigor of the thematic analysis is important in providing accurate and valid results in the qualitative analysis. The repetitive nature of thematic analysis provides richness to descriptions and a deeper understanding of each theme; the use of particular quotes from the focus group substantiate the findings relative to the research question [14].The use of selective coding allowed for identification of the over-arching themes related to perceptions and attitudes toward the chronic disease phenomenon. The use of several stages addressed reflexivity and recursiveness [14] [18]. Member checking was also used with several study participants further validating deducted themes [19].

There are four criteria to ensure trustworthiness of data: credibility, confirm ability, transferability, and dependability. Credibility was addressed during the focus group interview which took approximately $45-90$ minutes to ensure prolonged engagement with the participants. The prolonged interaction was designed to build trust and to provide an opportunity to identify distortions created by the participants or researcher that could affect interpretation of the data [20]. Merging of quantitative and qualitative data achieved triangulation of data. For peer debriefing, the study investigator met with an experienced researcher who was not associated with the research study to ensure that integrity of the process was maintained. In addition, the number of individuals involved as well as data saturation was used to achieve credibility in the study.

Dependability refers to the consistency of results over time. The use of multiple focus groups and consistent protocol use addressed dependability [10]. Confirm ability ensures objectivity and removes bias from the process. In order to establish both of these criteria, the use of an audit trail was utilized [10] and documented the research process and provide documentation of each step during the study. The audit trail included documentation of data collection, analysis, coding, and memos utilized during the study, as well as consistent documentation in field notes. Transferability was addressed through the use of purposive sampling in order to provide sufficient information so that the data could possibly be taken and used with other cases [10].

During the research process, bias was addressed. The researcher clarified personal beliefs and internal biases [21]. The nature of qualitative research places the researcher within the lived experience so that subjective bias is present. To minimize the possibility of bias, prior identification of beliefs and biases should be determined to increase the principal investigator's awareness.

\section{Results}

A total of 33 individuals expressed interest in the study and 29 were included by established criteria. Of the 29 participants in the focus groups, $76 \%$ were female $(n=22)$, $90 \%$ white $(n=26)$, and $55 \%$ had completed high school. A majority had been diagnosed with hypertension (83\%), followed by diabetes (31\%), and COPD (14\%). All participants had an average length of time since diagnosis of 10 years or greater (see Table 1 ).

Thematic Analysis

In reviewing the focus group interactions, two themes were most significant. Those themes were individualization and partnership. Individualization was seen in their perception of the disease experience; relationship with the healthcare provider; and interactions with the insurance health coach. Partnership was expressed as a desire to take more active role in healthcare decisions and increased their role as a team member. Something that at the time of the focus group, the participants felt was lacking in their care.

\section{Perceptions of Chronic Disease}

In each focus group, individuals were asked to describe how they felt about being diagnosed and how their lives had changes since diagnosis. Clearly, in the analysis of the themes, there were three distinct impressions that were consistent with diagnosis. These revealed three sub themes that supported the individuality of the disease phenomenon and how it is experienced. These sub-themes appeared to reflect the individual's perception of control in the disease process and awareness of the symptoms.

Hypertension

Those individuals who were diagnosed with hypertension expressed feelings associated with the most control over the disease process while at the same time expressing that the subtle symptoms allowed the individual to forget they had been diagnosed with hypertension.

Hypertension: Intangible 
Table 1. Means and standard deviations for demographic variables.

\begin{tabular}{|c|c|c|}
\hline Variable & $\mathrm{N}=$ number & Mean and Standard Deviation \\
\hline \multicolumn{3}{|l|}{ Gender } \\
\hline Male & $7(24 \%)$ & \\
\hline Female & $22(76 \%)$ & \\
\hline \multicolumn{3}{|l|}{ Race } \\
\hline White & $26(90 \%)$ & \\
\hline Native American or Alaskan Native & $2(6.8 \%$ & \\
\hline Spanish Ethnicity & $1(3.4 \%)$ & \\
\hline Age & & $\mathrm{M}=69.8(\mathrm{SD} \pm 8.3)$ \\
\hline \multicolumn{3}{|l|}{ Education } \\
\hline Completed High School & $\mathrm{N}=16(55 \%)$ & \\
\hline Completed College & $\mathrm{N}=13(45 \%)$ & \\
\hline \multicolumn{3}{|l|}{ Marital Status } \\
\hline Married & $\mathrm{N}=19(65.5 \%)$ & \\
\hline Single/divorced & $\mathrm{N}=6(20.7 \%)$ & \\
\hline Widow/Widower & $\mathrm{N}=4(13.8 \%)$ & \\
\hline \multicolumn{3}{|l|}{ Diagnosis } \\
\hline Hypertension & $N=24$ & \\
\hline Years since diagnosis & & $\mathrm{M}=22(\mathrm{SD} \pm 10.3)$ \\
\hline Diabetes Mellitus & $\mathrm{N}=9$ & \\
\hline Years Since Diagnosis & & $M=16(S D \pm 10.6)$ \\
\hline COPD & $\mathrm{N}=4$ & \\
\hline Years Since Diagnosis & & $M=10(S D \pm 13.49)$ \\
\hline Number of medications per day & & $\mathrm{M}=4.97(\mathrm{SD} \pm 2.54)$ \\
\hline Average annual physician visits & & M $3.07(\mathrm{SD} \pm 2.14)$ \\
\hline Time since last visit & 1 - 3 months & \\
\hline Health Rating & & $\mathrm{M}=3.13(\mathrm{SD} \pm 0.63)$ \\
\hline Very Good & $\mathrm{N}=6(8 \%)$ & \\
\hline Good & $\mathrm{N}=14(55 \%)$ & \\
\hline Fair & $\mathrm{N}=3(13.8 \%)$ & \\
\hline \multicolumn{3}{|l|}{ Physiologic Data } \\
\hline \multicolumn{3}{|l|}{ Blood Pressure } \\
\hline SBP & & $M=133( \pm 14.3)$ \\
\hline DBP & & $M=75( \pm 8.9)$ \\
\hline Oxygen Saturation & & $M=96.6( \pm 2.11)$ \\
\hline $\operatorname{HgbA1C}(\mathrm{n}=7)$ & & $M=7.0( \pm 0.59)$ \\
\hline BMI & & $\mathrm{M}=30.35( \pm 8.32)$ \\
\hline Shortness of Breath $(n=12)$ & & $M=2.75( \pm 0.45)$ \\
\hline
\end{tabular}

"You really don't see symptoms and it is hard to remember to take your medications.", "Having hypertension doesn't really affect me.", "Forget you have it; I can do just about anything I want too.", "Until you (researcher) said it, I really had not thought about it as a chronic condition."

Hypertension is seen to have few symptoms, manageable by medications, and least disruptive to their lives. As the individuals indicated the diagnosis of hypertension was not seen to have many effects on the individual and the impact was that the disease was not seen as a chronic condition that could cause multiple serious complications.

Diabetes

The perception of the diagnosis of diabetes was expressed differently by those in the group. The presentation of the disease was swift and the chronicity of the disease is readily apparent. The individuals verbalize that there 
was a constant awareness of the disease presence and the need to make changes was always foremost in the mind of the individual.

Diabetes: Unforgettable

It was a surprise, a shock, I was really anxious. You know I am going to have it forever. You are always aware. You have to make a lot of changes. It's not like you can forget you have it.

Most individuals in the focus groups expressed surprise at the suddenness and severity of the diabetes when initially diagnosed. The symptoms of the disease are prevalent and the care required was more disruption to their normal life pattern. But, the individuals were well versed in the disease process and felt that they had acquired sufficient knowledge to manage the disease.

With the diabetic, there was a consistent overshadowing of the possible complications.

"It's scary when you blood sugar drops. It's scary for you and your family. Your blood sugar is 40 and 340 and you wonder am I going to be able to do this?”

As suggested by this individual, the presence of diabetes and the adverse effects promoted a feeling of uncertainty and constant vigilance to prevent complications.

COPD

Those diagnosed with chronic obstructive pulmonary disease expressed a loss of control and a feeling of helplessness in the care process. Symptoms of the disease are significant in presentation. The disease was seen as causing limitations on daily activities of life.

Chronic Obstructive Pulmonary Disease (COPD): Daily Limitation

"It slows me down.”, "You just have to do what you can do and leave the rest.”, "My hypertension is controlled, but my COPD will never be controlled.”, "I battle it, it is always on edge.”, "I can’t do things like I used too.", "It's not that you can't control it, you can with medications and I do pretty good”, "If I am just sitting, I am fine .... I am perfect. But, if I try to do something then I get short of breath.”, "If it’s too cold, too hot, raining, ... a lot affects it”.

Those with COPD are acutely aware of the status of their breathing and confer significant restrictions on activities such as walking, exercising, and socialization. The perception was that it was well controlled as long as they could sit and not be short of breath and yet any normal daily activity was limited in that shortness of breath occurred with activation. With this disease, an attitude of acceptance toward what they saw as the inevitable outcome from an incurable disease, death.

"What good would it do to quit smoking now? In what years I have left, I won't see any reversal in the disease if I quit now. I will be dead before the changes take place." "But, you know it is never going to get better, there's no cure."

There was a perceived helplessness in this group resulting from the understanding that the disease process was a continual downward progression and that maintaining the best control they can was the focus of management.

The phenomenon of the disease experience is individualized as seen in the focus group discussions. The disease experience varies from barely being aware of the diagnosis to being very limited in basic daily functioning causing a significant impact on quality of life.

In looking at the perception of chronic disease, an overarching theme of individuality reigns as each individual expresses the personalized impact the disease has on one's life.

Perceptions towards Making Health Behavior Changes

As the attitude toward the actual disease process varies, the analysis also demonstrated a variety of attitudes that varied by individual. Each focus group was asked to describe how they felt about making changes as a result of being diagnosed. These changes included diet and exercise as well as medication management. The responses discussed making changes, and also focused upon motivators and de-motivators of change.

"I am just grateful they have medications for it." "At first I didn't make any changes, I was probably in denial.". "I want to lose weight." "If I lost forty pounds, I don’t think I would have to take blood pressure medications.", "I have always been very active, so up until this point I have not had to exercise." "I try to be compliant, but there are things I am not compliant with like exercise. I just hate to exercise." "It's hard, 
it's not like you can quit eating (diabetic).”

As the themes were further explored subcategories of themes were identified. Motivators for change could be further divided into internal and external foci that motivated change. A motivating internal focus for change focused on the rewards that an individual could receive if changes were successfully maintained and maintaining quality of life.

\section{Internal Foci:}

"Encouraged when you see the changes work (decreased weight, blood sugar, blood pressure).", "I like feeling good. I want a good quality of life. I don't want to be put into a nursing home.", "Seeing others having complications, I don’t want to end up like that.”

\section{External foci:}

"I want to be able to do things with my family.", "My husband is very active and I want to be able to keep up with him.", "My grandchildren, I want to be around to see them grow up.", "I want to be able to do things with my grandchildren.”, “I want to be able to go to church. If I can't go to church, you might as well bury me.", "I want to see my grandchildren graduate, get married and have babies."

In this study, the older adult with chronic disease focused not only on longevity, but on quality of that longer life. Reasons expressed that encouraged the individuals to make change, centered on spousal/partner and the family interactions. Not only were the interactions important but the quality of those interactions were significant to the individuals and supporting the theme of each person having their own motivation for changing and should be seen as an individualized process. The individuals saw themselves as continually being an active participants in future relationships if successful health behavior change was to be made.

In changing behaviors, the emphasis on individualization was found when an individual was learning about or attempting change. Whether based on disease process or level of learning, individuals felt that being recognized as a person and not a disease was important.

Not only were the groups asked about current relationships, they were asked to visualize a situation in which they were required to change and to describe what format that they would most likely participate in. The types of social support that they preferred to help with change varied. The groups were asked about how they would be most likely to initiate a health behavior change and the methods of change varied among individuals as well:

"I couldn’t keep up with a support group.”, "It depends on the support group as to whether I would attend.", "I think coaching would be ok.”, "Yea, having a coach would be good.”, "I don’t think I would like internet. I don't know much about the internet. I would have to look at it.", "I like to do things with (spouse), we do pretty good together.", "It makes it better to have someone to do it with.", "I've taken classes, but it is hard when you don’t know anyone and if your late you are definitely not going to go in.”

Each individual had different concerns and needs to be addressed in the health behavior change process. In making changes in behaviors to manage the chronic disease process, the individuals felt that family was most preferred while coaching was also of interest to them.

At the same time, the participants recognized that there were de-motivators for behavior change. The reasons for being unable to make behavior changes consistently varied and were based upon either physical limitations or psychosocial issues. Physical de-motivators tended to be consistent within the groups and reflected interference or impedance caused from pain or complications of another disease. The psychosocial barriers were related to loneliness and self-consciousness. These conversations took place using exercise and diet the behavioral changes needed for successful self-management.

While many understood that their disease management required them to incorporate exercise into the plan of care, many expressed a reluctance or inability to exercise.

“I have COPD, I can’t exercise. I would like to lose weight, but I can’t do those things.”, "I can’t because of my COPD. If it's humid, hot, or too cold, it makes my breathing worse.”, "I can't because of my pain (arthritis).", "I hate to exercise, I just hate it. You get sweaty and have to change your clothes, makeup, hair... I just hate it. I know it works, but I am just as glad when it's over.”

For those who participated in organized classes, lack of knowledge of other participants deterred participa- 
tion.

"You know if you go to a class and you don't know anyone its hard or if you're late, you sure are not going to go in there."

The lack of rewards for maintaining the behavior change was also hindrance to behavior change.

“It's unfair I can exercise and do all the right things and I don't get the results that others do. It's frustrating.”, "It would be easier if you saw some kind of reward." "Well it's hard because they keep changing the goal. You work to get our blood pressure down to where they want it and then they change it on you."

For dietary changes, psychosocial factors were prevalent from self-consciousness in not eating foods that were not on their diet or loneliness.

“It’s hard eating for one.”, “You can’t cook for just one person. I don’t sit at the table if it’s just me. I just don't like to sit there by myself.”, "I get self-conscious when I am eating with others...what if I offend someone by not eating what they have fixed. They don't understand I may not be able to have it. I just do the best I can, but I hate to offend them.”

Psychosocial and physical barriers were prevalent in the conversations. These were all cited as reasons that would prevent an individual for initiating and maintaining an exercise routine or maintaining diet changes. A need to see improvement based upon the behavior change was measures in blood pressure, weight, or blood glucose values by the participants relative to their respective diseases. The individuals with COPD saw little opportunity to make changes based upon the limitations of their disease.

"I don’t know what I could do to control it (COPD).”

In looking at each person's perception towards the disease process and behavior change, it is determined that each disease is inherently different in the impact on an individual's life.

Partnership with Healthcare Providers

Having established that individuality is important in the chronic disease process, another theme became prevalent during analysis. The person not only wants to be treated as an individual, but also as an equal member, a partner, in the care process. The relationship with the provider was seen as a support mechanism in which partnership was considered important and perceived lacking by the participants. As for the provider, the participants expressed trust and satisfaction with the level of knowledge of the provider, but greater dissatisfaction with the lack of time, lack of input into goal setting, and quality of the relationship.

The participants described the current relationship as one with parental overtones.

“He'll fuss. I don't want to be fussed at.”, "He fussed at me the last time I was there too”, "I lost weight and my blood pressure got low. So, I stopped taking my medicine, but I didn’t tell my doctor.”, “Mine fussed at me and I finally told him that when I got ready to talk about it (smoking) I would let him know. Otherwise he was not to bring it up again.”

The participants are reluctant to engage the provider to avoid being treated as if they have done something wrong.

"I am heading him off today and presenting him with what I see as the plan.”

A plan of action is seen as an appropriate mechanism for changing the healthcare providers' response to their visit. At the same time, the participants felt that there were other inhibitors to the interaction.

"Not like it used to be, they used to know you and your family.”, "Time is limited to answer your questions.”, “They only have so much time, who is going to answer your questions?”, "I feel sorry for them because they have only a certain amount of time to spend with you, but it's their job to answer my questions.”, "I trust him. He provides good care and is very knowledgeable.", "He should send me to someone who can answer my questions.”, "I want someone, a coach, to teach me what I need to know.”, "I want a partnership, I don’t want to be fussed at.”, "I am the one taking care of this every day.”.

The care of the disease process is a team approach in which the individual is the center. The results of the analysis demonstrated that older adults want to work together with their provider, not be told what to do and not 
be "fussed at" as if a small child. The individuals wants input and to be heard in the decision making and care process recognizing that they will be the one actively addressing change.

The participants also expressed the need to have the opportunity to ask questions and to improve their knowledge about the disease process.

"I am on this new medicine. They gave me a brochure and a DVD. That was all good, but who is going to answer my questions?", "I need someone who will teach me! What do I do if my blood sugar drops? I need to know what to do.", "When I was diagnosed they sent me home with insulin. Insulin! I didn’t know anything about it, I just wanted to cry.", "I have diabetes, but I would like to lose weight. I know it’s hard to and I told my doctor that I wanted to lose weight. He looked at me and said, 'well go ahead.' Now what kind of help is that?" "I need somebody, who will teach me what to do".

Acquiring knowledge seems to be lead predominately by those with diabetes who have an increased number of activities to complete to manage the disease process. Those with hypertension expressed interest in more education, while those who were diagnosed with COPD had no input into the conversation.

An unexpected finding in the conversation was revealed in the evolution of the discussion. Most participants voiced similar opinions towards the current status of health insurance and the use of what is being called "the health coach". The insurance health coach is someone who calls based upon screening of health documents. The content of the call can vary from individual to individual based upon the insurance companies chosen focus. The perception of the "health coaching" role in self-management was perceived negatively.

"I am so angry (slams hand down on table).", "The insurance company is telling me what to do and if I don't... what are they going to do? Raise my premiums.", "They have this health coach call you and they ask you questions. It's not individualized, it's based upon your disease.". "Everybody treated the same.”, "It's a waste of time, what are they going to tell me that I don't already know?" "The person is simply asking a question that is on the computer screen.", "I am not going to let the insurance company (health coach) get credit for what I am doing. I got my shot, and they check a box off and they get credit for me doing it.”, "In some cases, I know more than they do. They can't even tell me why the insurance company flagged me.", "I asked a question and they tell me that they will have to find someone who can help me.", "they were at least trying to help me, but shouldn't someone know about my condition?”, "I don't have time to sit there and chit chat. It makes you... I am sitting there smoking and I wouldn’t be if they would get off there.”

Individuals with chronic disease, expressed frustration over being required to participate in a process in which there appears to be no inherent benefit to them (other than keeping their premium down) and one in which they have no input. The insurance company's utilization of the "health coach" is perceived as a waste of time and of little benefit to the individual.

\section{Discussion}

Self-management is an important method of managing chronic disease, but over time self-management programs have not sustained lasting change. Most programs are group activities that may be beneficial for some, but as found in this qualitative study not all individuals may respond the same to behavior change intervention.

For those individuals participating in the study, two overall themes could be identified individualism and partnership. Each person described a unique experience with the chronic disease process and a preference for a treatment plan that was individualized to their needs. A person with chronic disease is still an individual and does not want to become simply the face of a specific disease. Partnership was a prevalent theme with the participants verbalizing the desire to take a more active role and have a voice in healthcare decisions. The clients desired to have the team work together to develop goals that not only addressed the disease, but allowed them to meet their own personal goals. The older adult recognized that ownership of the disease rests with them and that the services of the healthcare provider should be driven by the needs of the consumer.

For health behavior change to occur, an individual must participate in setting goals, while addressing barriers to change. In these focus groups, a desire to be treated as a member of the team and develop mutually agreeable goals seems to be at the forefront of concern. At present, as perceived by the participants, the locus of control in the self-management process is driven by healthcare providers and health policy (Figure 1), yet the older adult desires to have individualized relationship that is in partnership with the treatment team (Figure 2). 


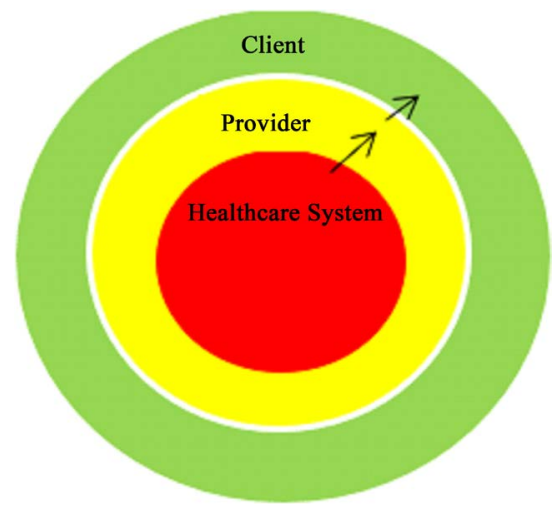

Figure 1. Locus of control in health management as perceived by adults.

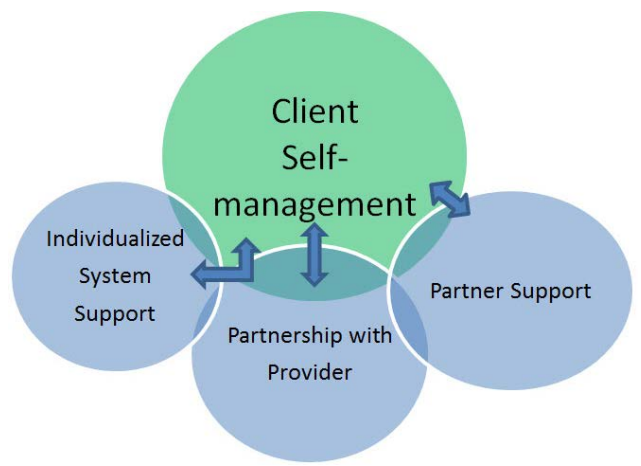

Figure 2. Older adult desired health management model.

They do not want to be told what to do... they want to be asked what they would like to do. In most aspects of life, an individual has the right of opinion, right to know, and autonomous decision making and yet in chronic disease management, healthcare is falling short of meeting the needs of the consumer. As seen in this study, many felt that their educational needs were not being met and expressed a desire for increased education and opportunities to ask their specific questions from someone knowledgeable in the disease process. Figure 2 shows a self-management model in which the stakeholders in the process have an overlapping and reciprocal relationship. The client, provider, partner, and health entities work together with the central focus being the older adult and their desire for health behavior change.

Inclusion of social support was a subtheme of the focus group discussions and evolved around the important of social support systems. Spouses/significant other appeared to be most likely utilized as a support system in the change behavior process, but as the discussion evolved the healthcare provider and insurance support was highlighted. Little information was uncovered regarding the role of children in social support, but the role of spouses/significant other and close friends appears to influence an individual's attitude towards health behavior change. Of those couples that participated in the study, all verbalized strong spousal/significant other support with medication, diet, and exercise change. There was an overall feeling that having good spousal participation was beneficial while one individual shared a lack of spousal/significant other support and reflected on preferring a greater amount of support to be successful.

The barriers and motivators to health behavior change were also individualized as to what could help them be most successful in making health behavior change and also to help healthcare providers understand that non-adherence may not simply be a matter of refusal but may be motivated by physical or psychosocial barriers such as pain and loneliness. Many of the participants expressed an interest in making changes, yet could not in the face of barriers.

\section{Limitation of Study}

Sampling for the study used purposive sampling which limits generalizability of findings to the population. The 
focus groups were held in faith based organizations and most individuals already knew each other prior to participation. The composition of the focus group could have altered responses to socially appropriate answers. In addition, the severity of illness and number of chronic diseases diagnosed could also have impacted the individual response.

\section{Conclusion}

Results of this qualitative descriptive study indicate that there is a disconnect between the perceptions of the older adult towards chronic disease care and behavior change in comparison to what is presently being experienced in healthcare interactions. This disconnect suggests that further research regarding healthcare providers' attitudes towards the older adults' role in chronic disease care should be explored. The preference for older adults to include spouses in the change process or immediate family suggests that developing a plan that includes this support system may be beneficial. The themes of individualization and partnership suggest that a method of health behavior change that addresses these two main concerns should be explored to enhance the older adult's satisfaction and success in chronic disease care. Understanding the perceptions of the older adult towards the chronic disease phenomenon can provide healthcare providers with insight that can be valuable in providing specific care to this unique population.

\section{References}

[1] Centers for Disease Control and Prevention (2013) The State of Aging \& Health in America. C.f.D. Control, Editor, US Department of Health and Human Services, Atlanta.

[2] Whittemore, R. and Dixon, J. (2008) Chronic Illness: The Process of Integration. Journal of Clinical Nursing, 17, 177187. http://dx.doi.org/10.1111/j.1365-2702.2007.02244.x

[3] Centers for Disease Control (2009) Chronic Disease: The Power to Prevent, the Call to Control. U.S.D.o.H.a.H. Resources, Editor, Atlanta.

[4] Centers for Medicare and Medicaid Services (2010) National Expenditure Projections 2010-2020: Forecast Summary. Centers for Medicare and Medicaid Services, Woodlawn.

[5] Dunn-Cane, K., Gonzalez, J. and Stewart, H. (1999) Managing the New Generation. American Journal of Operating Room Nurses, 69, 930-940. http://dx.doi.org/10.1016/S0001-2092(06)62292-8

[6] Phillipson, C., et al. (2008) Social and Cultural Constructions of Ageing: The Case of the Baby Boomers. Sociological Research Online, 13, 5. http://dx.doi.org/10.5153/sro.1695

[7] Bandura, A. (2004) Health Promotion by Social Cognitive Means. Health Education \& Behavior, 31, 143-164. http://dx.doi.org/10.1177/1090198104263660

[8] Glanz, K., Rimer, B.K. and Viswanath, K., Eds. (2008) Health Behavior and Education. 4th Edition, Jossey-Bass, San Francisco.

[9] Bandura, A. (1986) Social Foundation of Thoughts and Action: A Social Cognitive Theory. Prentice-Hall, Inc., Upper Saddle River.

[10] Polit, D.F. and Beck, C.T. (2012) Nursing Research: Generating and Assessing Evidence for Nursing Practice. 9th Edition, Lippincott, Williams \& Wilkins, Philadelphia.

[11] Krueger, R.A. (2009) Focus Groups: A Practical Guide for Applied Research. Sage Publications, Thousand Oaks.

[12] Sandelowski, M. (2000) Combining Qualitative and Quantitative Sampling, Data Collection, and Analysis Techniques in Mixed-Method Studies. Research in Nursing \& Health, 23, 246-255. http://dx.doi.org/10.1002/1098-240X(200006)23:3<246::AID-NUR9>3.0.CO;2-H

[13] Tennessee Department of Health (2008) Tennessee Deaths 2007.

[14] Braun, V. and Clarke, V. (2006) Using Thematic Analysis in Psychology. Qualitative Research in Psychology, 3, 77101. http://dx.doi.org/10.1191/1478088706qp063oa

[15] QRS International (2012) NVivo. http://www.qsrinternational.com/products.aspx

[16] Sandelowski, M. and Leeman, J. (2012) Writing Usable Qualitative Health Research Findings. Qualitative Health Research, 22, 1404-1413. http://dx.doi.org/10.1177/1049732312450368

[17] Plano Clark, V.L. and Creswell, J.W. (2008) The Mixed Methods Reader. Sage Publications, Thousand Oaks.

[18] Fereday, J. and Muir-Cochrane, E. (2006) Demonstrating Rigor Using Thematic Analysis: A Hybrid Approach of Inductive and Deductive Coding and Theme Development. International Journal of Qualitative Methods, 5, 80-92. 
[19] Creswell, J.W. and Plano Clark, V.L. (2011) Designing and Conducting Mixed Methods Research. 2nd Edition, Sage Publications, Los Angeles.

[20] Lincoln, Y. and Guba, E. (1985) Naturalistic Inquiry. Sage Publications, Newbury Park.

[21] Specialze, H.J. and Carpenter, D.R. (2007) Qualitative Research in Nursing. 4th Edition, Lippincott, Williams \& Wilkin, Philadelphia. 


\section{Appendix 1}

Focus Group Questions

1. Each one of you has been diagnosed with a chronic illness such as hypertension, Chronic Obstructive Pulmonary Disease, or Diabetes. When you think about what has happened since you were diagnosed, can you tell me.... What is it like living with (COPD, HTN, DM)?

2. When you were diagnosed you were asked to make changes so that you could better control you illness. Can you describe.

How do you take care of your (COPD, HTN, DM)?

3. When you think about what has happened since you diagnosis, can you tell me.....

a. What changes have you had to make in your everyday life since diagnosed with (COPD, HTN, DM)?

You have described some changes.......

b. How do you feel about those changes that you had to make?

4. What things could you do better to manage your (COPD, HTN, DM)?

5. In looking at your health and diagnosis,

What keeps you from managing these better?

6. When you think about your health,

What are you doing well in managing your (COPD, HTN, DM)?

How do you feel about the things that you still need to change?

7. In looking at having a chronic illness.......

What motivates you to make change and manage your (COPD, HTN, DM)?

8. In order to take care of yourself and manage your illness, .....

a. can you tell me how you feel about setting goals? Do you?

9. If you do set goals or thought about setting goals, describe.

b. How do think that you setting goals would or does impact managing your illness?

c. Can you give me an example of goals you set?

10. Thinking about the things that you have been asked to do related to taking care of yourself and your illness, What could you use more help with in managing your illness?

In managing your illness, it is important to have support.

Can you describe the support that you have with taking care of your illness?

Who could help you more with managing your illness? (family, friends, nurse, doctor)

11. In thinking about improving the support you get,

How do you feel that they could give you more support?

12. If the amount of support you had improved,

What might be some benefits to you with getting more support to manage your illness?

13. There are different ways to offer support for your illness from a healthcare provider,

Can you describe.

How do you feel about the different ways of support to manage your illness and make change such as groups, (support) internet, (information or education modules) and working with a health coach? (one on one meeting) 